\title{
Integrable Turbulence and Rogue Waves: Breathers or Solitons?
}

\author{
J. M. Soto-Crespo, ${ }^{1}$ N. Devine, ${ }^{2}$ and N. Akhmediev ${ }^{2}$ \\ ${ }^{1}$ Instituto de Óptica, CSIC, Serrano 121, 28006 Madrid, Spain \\ ${ }^{2}$ Optical Sciences Group, Research School of Physics and Engineering, \\ The Australian National University, Canberra ACT 2600, Australia
}

(Received 30 September 2015; published 9 March 2016)

\begin{abstract}
Turbulence in dynamical systems is one of the most intriguing phenomena of modern science. Integrable systems offer the possibility to understand, to some extent, turbulence. Recent numerical and experimental data suggest that the probability of the appearance of rogue waves in a chaotic wave state in such systems increases when the initial state is a random function of sufficiently high amplitude. We provide explanations for this effect.
\end{abstract}

DOI: 10.1103/PhysRevLett.116.103901

Turbulence is an irregular behavior of dynamical systems characterized by chaotic changes of the flow parameters [1]. It has attracted much attention in relation to integrable and Hamiltonian systems [2-7]. The knowledge of its properties is vital for oceanographic [8] and atmospheric [9] studies as well as for optical communications [10]. Although the equations that describe wave propagation in these fields are not integrable, in a first approximation they are close to them [8]. Of special interest is the rogue wave (RW) content of a turbulent wave field, represented by the extreme deviations from the average wave amplitude [2,3]. There are several crucial results on this subject [3-7], but many questions remain. One of the interesting results to be mentioned is that Peregrine breathers are not the highest waves that may appear in a chaotic wave field [2]. This had already been observed in previous numerical simulations [11], but its importance had not been fully recognized until recently [2]. The highest waves appear as a result of superpositions of breathers and solitons [11] rather than as a single Peregrine breather as conjectured in Ref. [12].

Another paramount finding is that the probability of RW appearance depends very much on the initial conditions [3]. One of the mechanisms that contributes to the formation of a chaotic wave field is modulation instability (MI) [13]. MI transforms an initially smooth function into a chaotic wave field. A chaotic wave field can also be created directly using an initial condition with high amplitude chaotic components. In integrable and Hamiltonian systems this chaotic field contains solitons, justifying the term "soliton turbulence" suggested in Ref. [14]. The relative contribution of each mechanism is not easy to find when the field is already highly turbulent. However, it can be found for initial conditions consisting of a continuous wave (cw) component mixed with noise of variable amplitude. Small amplitude noise leads to modulation instability of the $\mathrm{cw}$ while large amplitude noise makes a direct contribution to the soliton content. A significant difference in the probability of appearance of rogue waves in these two cases was observed experimentally in Ref. [3], and in numerical simulations in Ref. [15]. Both simulations and experiment show that the probability of RW generation increases with the increase of the initial noise level. However, clear explanations for this phenomenon have not been given. In the present work, we provide explicit clarification for this remarkable effect.

In order to find the mechanism of the emergence of a higher number of rogue waves, we have performed numerical simulations with the dimensionless nonlinear Schrödinger equation (NLSE),

$$
i \psi_{\xi}+\frac{1}{2} \psi_{\tau \tau}+|\psi|^{2} \psi=0
$$

taking as the initial condition a constant background of unit amplitude with random perturbation on top of it. Namely, as in Ref. [15-17], we used the function $\psi(\tau, 0)=1+\mu f(\tau)$, where $f(\tau)$ is a normalized complex random function whose standard deviation is $\sigma=1$. When multiplied by the variable coefficient $\mu$, this gives the standard deviation for the function $\psi$. The real and imaginary parts of the function $f(\tau)$ are taken as Gaussian distributed and Gaussian correlated functions [18], with variable correlation length $L_{c}$. Real and imaginary parts of the random field are mutually uncorrelated functions. The parameter $\mu$ that we varied in the simulations is related to the variance of the resulting initial field intensity distribution $\sigma^{2}=\left\langle I^{2}\right\rangle-\langle I\rangle^{2}$, where $I=|\psi|^{2}$, and \langle\rangle stands for the mean value in $\tau$ over thousands of realizations. The use of two variable parameters, $\sigma$ and $L_{c}$, allowed us to reveal a more complex dynamics of integrable chaos than in previous works.

Figure 1 illustrates the main features of the phenomenon. Figure 1(a) shows the intensity profile of three typical initial conditions used in our simulations. Only a small part of the entire $\tau$ interval is shown here. The value of the mean field intensity $\langle I\rangle$ is taken always equal to 1 . The value of $\sigma$ for each case is given inside the figures. The correlation 

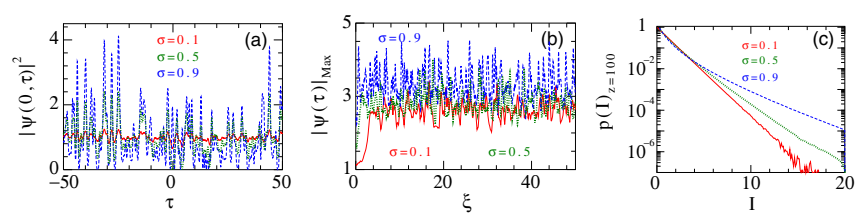

FIG. 1. (a) Initial field intensity for three values of $\sigma$. The solid red curve corresponds to a small perturbation, $\sigma=0.1$, the dotted green curve is for moderate perturbation, $\sigma=0.5$, and the dashed blue curve stands for the highest perturbation, $\sigma=0.9$. The correlation length $L_{c}=0.76$. (b) Evolution of the maximum of the field amplitude versus $\xi$ for the above three realizations of the initial field. (c) The probability density function for the field intensity at $\xi=100$ for the same values of $\sigma$.

length $L_{c}$ in this case is 0.76 . The solid red curve that corresponds to $\sigma=0.1$ shows relatively small random deviations from the $\mathrm{cw}$ with amplitude 1 . The dotted green curve that corresponds to $\sigma=0.5$ shows higher chaotic deviations around the $\mathrm{cw}$, while the blue dashed curve that corresponds to the highest $\sigma=0.9$ shows a considerable amount of noise. The cw component in this last case is completely out balanced by noise.

Figure 1(b) illustrates the result of the simulations with the above initial conditions. It shows the peak amplitude (absolute maximum of $|\psi|$ over the whole $\tau$ interval of simulation) as a function of $\xi$. As expected from the theory of modulation instability, for the lowest values of $\sigma$, the maximum increases exponentially at the initial stages of evolution. It evolves chaotically after the saturation is reached at a certain propagation distance. The convergence to a chaotic state is similar but quicker when $\sigma=0.5$. For $\sigma=0.9$, the evolution is chaotic from the very beginning. The plots in Fig. 1(b) are not necessarily smooth. The location of the peak amplitude in $\tau$ may change abruptly, especially for a wide numerical grid.

A remarkable fact observed in Fig. 1(b) is that the maximal field amplitude takes higher values for higher $\sigma$. Namely, in the case of $\sigma=0.9$, the highest amplitudes are roughly 1.5 times higher than in the case of $\sigma=0.1$. Each of the above examples represents only one, randomly chosen, realization. In order to calculate the statistical characteristics of the phenomenon, we repeated the simulations with thousands of different, but statistically equivalent, initial conditions. Figure 1(c) shows the probability density function (PDF) of the field intensity obtained after propagating a distance of 100 units along the $\xi$ axis. The PDF's curves are not settled at the initial stages of propagation, but converge to a nearly stationary regime and practically do not change after $\xi=20$. The curves in Fig. 1(c) show clearly that the tails of the PDF increase considerably with $\sigma$. At $I \approx 16$, the probability increases roughly 20 times when $\sigma$ increases from 0.1 to 0.5 , and 20 times more when it increases from 0.5 to 0.9 . Thus, the probability of generating rogue waves is significantly enhanced when the chaotic component in the initial conditions is comparable or stronger than the cw. As the total energy density in the initial conditions is kept the same in all three cases, the origin of the enhancement has to be sought for in the internal dynamics of the system governed by the NLSE rather than in the changes of energy.

In order to find the reason for this enhancement, we used the fact of the integrability of the system. Namely, the NLSE originates from the compatibility condition of the following set of linear matrix equations [19]:

$$
\begin{gathered}
R_{\tau}=U R+\lambda J R, \\
R_{\xi}=\left(\lambda^{2}+\lambda U+V / 2\right) R,
\end{gathered}
$$

where the $2 \times 2$ matrices $U, V$, and $J$ are given by

$$
U=\left(\begin{array}{cc}
0 & i \psi^{*} \\
i \psi & 0
\end{array}\right), \quad V=\left(\begin{array}{cc}
-i|\psi|^{2} & \psi_{\tau}^{*} \\
-\psi_{\tau} & i|\psi|^{2}
\end{array}\right), \quad J=\left(\begin{array}{cc}
i & 0 \\
0 & -i
\end{array}\right),
$$

while

$$
R=\left(\begin{array}{l}
r \\
s
\end{array}\right)
$$

is a column matrix composed of the two complex functions $r(\xi, \tau)$ and $s(\xi, \tau)$.

One of the major results of the inverse scattering technique [19] is that the spectrum of eigenvalues of Eq. (2) does not depend on $\xi$. This means that the dynamics of the field $\psi(\xi, \tau)$ at any $\xi$ is defined by the spectrum of the eigenvalues of Eq. (2) at $\xi=0$, i.e., defined by the initial condition. The latter plays the role of a potential in the eigenvalue and eigenfunction problem. By solving Eq. (2) for each initial chaotic "potential" $\psi=\psi(\xi=0, \tau)$, we can find the set of eigenvalues $\lambda$ and eigenfunctions $R$ of this problem. These eigenfunctions are either nonlocalized solutions that correspond to breathers and radiation waves or functions localized on the "potential wells" created by the holes of the chaotic function $\psi$. The latter correspond to solitons. The complete sets of eigenvalues $\lambda$ are of crucial importance for finding the reasons for the enhancement of the probability of the appearance of rogue waves.

We have numerically solved the eigenvalue problem Eq. (2) using the initial chaotic functions $\psi$ for the same three values of $\sigma$ as above. The sets of complex eigenvalues $\lambda$ found this way are shown in Fig. 2. The results for various chaotic functions generated in each realization differ. However, the average location of the eigenvalues occupies an area in the complex plane well determined by the corresponding values of $\sigma$. The three plots in Fig. 2 show the result of the superposition of 11 realizations. In all cases, there is a continuous line of eigenvalues located on the real axis that correspond to radiation waves. They extend to much larger values along the horizontal axis of $\operatorname{Re}(\lambda)$ than those shown in the figure. 


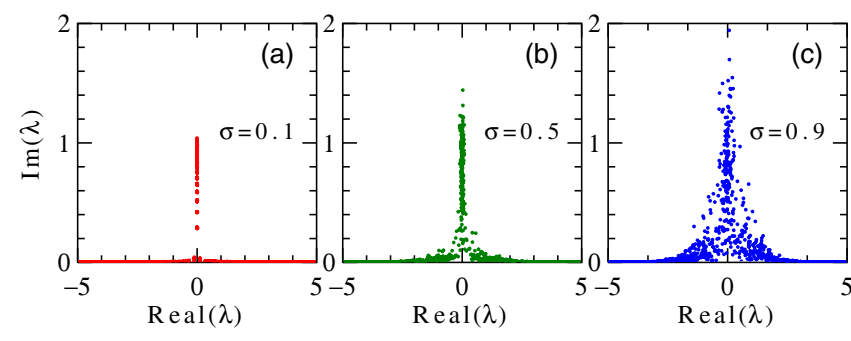

FIG. 2. Complex eigenvalues $\lambda$ calculated for the initial conditions $\psi(0, \tau)$, with (a) $\sigma=0.1$, (b) $\sigma=0.5$, and (c) $\sigma=0.9$. As the eigenvalues appear in complex conjugate pairs, only the upper half of the complex plane is shown.

When $\psi(\xi=0, \tau)=$ const, the eigenvalues located along the vertical axis correspond to Akhmediev breathers (AB). Figure 2(a) shows the set of eigenvalues for $\sigma=0.1$, which gives an initial $\psi$ function close to the unperturbed cw. Smaller perturbations produce practically the same spectrum (and, accordingly, identical PDFs). The upper limiting point $\lambda=i$ in the spectrum corresponds to the Peregrine breather. The latter has a peak amplitude of 3 , which is the maximum possible for breathers. The eigenvalues located below the point $\lambda=i$ on the imaginary axis correspond to the excitation of $\mathrm{ABs}$ which have lower amplitudes. They continuously occupy the whole interval of unstable frequencies inside the modulation instability gain curve. Discreteness in Fig. 2(a) is due to the use of periodic boundary conditions in our numerical scheme. Increasing the number of mesh points leads to denser distribution of eigenvalues along the vertical axis.

Figure 2(b) shows the set of eigenvalues obtained when the chaotic perturbation is larger, $\sigma=0.5$. The spectrum becomes visibly chaotic. Most of the excitations are ABs, although some of them may split into solitons. As we know from the work by Mahnke and Mitschke [20], small perturbations do not destroy Akhmediev breathers if the governing equation remains integrable. However, not all eigenvalues remain strictly on the imaginary axis. Those eigenvalues that acquire a real part correspond to solitons. Moreover, the eigenvalues that previously were located on the real axis and described radiation waves now have a finite imaginary part and also correspond to solitons. The eigenvalues with imaginary part exceeding $i$ may correspond to Kuznetsov-Ma solitons [2]. As can be seen in Fig. 2(b), there is a comparable number of breathers and solitons in this case.

Larger deviations from the $\mathrm{cw}$ in the initial conditions result in practically no eigenvalues left exactly on the imaginary axis. Figure 2(c) shows the set of eigenvalues obtained when $\sigma=0.9$. The total number of eigenvalues remains the same as before. Practically all eigenvalues now correspond to solitons rather than breathers. Their amplitudes are twice the imaginary part of the eigenvalue. They acquire velocity, which is defined by the real part of the complex eigenvalue. These solitons are located on a background that consists of a sea of radiation waves. The $\mathrm{cw}$ that was present in the initial conditions does not exist anymore. Thus, when the perturbation is comparable to the amplitude of the initial $\mathrm{cw}$, the continuous spectrum of breathers is replaced by a spectrum of solitons. Figure 2(c) shows clearly such transformation.

As the spectrum of eigenvalues remains unchanged on propagation, the field evolution is defined by the relative number of solitons and breathers in the chaotic wave field. Rogue waves are the result of the interaction of either breathers or solitons with each other. The switch of the wave content from breathers to solitons changes the chaotic pattern dramatically. Examples of these patterns are shown in Fig. 3. Namely, Fig. 3(a) shows the field amplitude $|\psi|$ for $\sigma=0.1$. The pattern corresponds to the interaction of a multiplicity of breathers. In contrast, Fig. 3(c) shows the case when $\sigma=0.9$. The pattern here is dominated by solitons and their collisions. In the intermediate case, $\sigma=0.5$ shown in Fig. 3(b), both breathers and solitons are present. The highest amplitudes in these patterns are accented in red. These amplitudes differ as shown in the caption of Fig. 3. The corresponding peak intensities $I=|\psi|^{2}$ are around $I=9.0$ in Fig. 3(a), above 12.25 in Fig. 3(b). and above 16 in Fig. 3(c). These figures show clearly that solitons create the highest amplitude waves in the chaotic pattern.

The amplitudes that appear in the chaotic patterns are in agreement with the eigenvalue analysis. The highest amplitude of the breather family, which is 3, appears in the case of the Peregrine breather with eigenvalue $i$. This is the lower threshold of high amplitudes observed in Fig. 3(a). On the other hand, the imaginary part of the soliton eigenvalues in Fig. 2(c) reaches the value of almost 2.0, which corresponds to a soliton amplitude of 4.0. This is also in agreement with observations in Fig. 3(c). These amplitudes are higher than the amplitudes of breathers. Peak amplitudes at soliton and breather collision points may reach even higher values, thus causing the generation of rogue waves. Another important feature of the patterns in Fig. 3 is the fact that the solitons and breathers appear on top of a multiplicity of radiation waves. This can be seen more clearly in the 3D plots of Fig. 4, which show

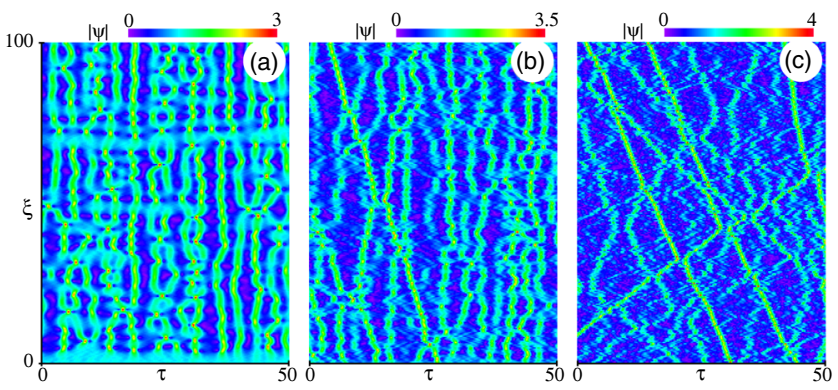

FIG. 3. Chaotic patterns generated for (a) $\sigma=0.1$, (b) $\sigma=0.5$, and (c) $\sigma=0.9$. Red spots in these patterns correspond to amplitudes higher than 3.0 in (a), 3.5 in (b), and 4.0 in (c). 


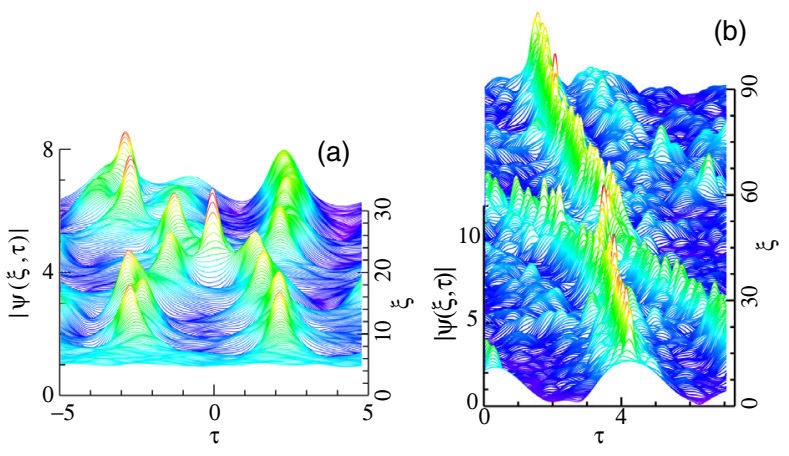

FIG. 4. Parts of the chaotic patterns of Fig. 3 shown as 3D plots for (a) $\sigma=0.1$ and (b) $\sigma=0.9$. The background consists of a multiplicity of radiation waves causing the amplitudes of breathers in (a) and solitons in (b) to evolve randomly.

smaller parts of the chaotic fields in Figs. 3(a) and 3(c). In particular, this superposition leads to irregular changes of the breather and soliton amplitudes which are well visible in Fig. 4.

Another factor that plays a significant role in this chaotic evolution is the correlation length of the initial random field. The smooth or sharp changes of the initial field influences the number and parameters of the solitons generated at the beginning of the process and therefore determines its corresponding PDFs. Figure 5(a) shows the intensity variations in the initial conditions with fixed $\sigma=0.5$ for three different correlation lengths. The smallest correlation length $L_{c}=0.19$ leads to a fast changing function while the largest $L_{c}=1.8$ results in slower variations.

Figure 5(b) shows the sets of eigenvalues calculated for the same $\sigma$ and correlation lengths as in Fig. 5(a). This figure shows that for the lowest correlation length $L_{c}=0.19$ (red triangles), the maximum value of the imaginary part of all the eigenvalues does not exceed 1 . This means that the soliton amplitudes are smallest among the three cases. The soliton amplitudes are higher when $L_{c}=0.76$ and the highest when $L_{c}=1.8$. A similar distribution of eigenvalues is observed in the case of $\sigma=0.9$ shown in Fig. 5(c) except that the soliton amplitudes and velocities take even larger values, producing a broader distribution of eigenvalues around the vertical axis. When $\sigma=0.1$, the eigenvalue spectrum is practically

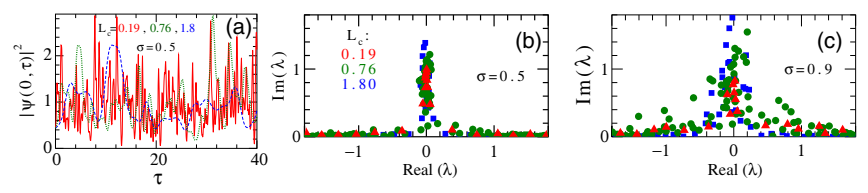

FIG. 5. (a) Examples of initial field intensity for fixed $\sigma=0.5$ and three sizes of the correlation length $L_{c}$. The solid red curve corresponds to $L_{c}=0.19$, the dotted green curve to $L_{c}=0.76$, and the dashed blue curve stands for $L_{c}=1.8$. (b) Sets of eigenvalues for the same three correlation lengths. (c) Sets of eigenvalues when $\sigma=0.9$. independent of the correlation length and similar to the one shown in Fig. 2(a).

The above results allow us to anticipate the influence of the correlation length on the tails of the corresponding PDFs. Each individual plot for a particular $\sigma$ in Fig. 6 shows three curves for three different correlation lengths. When $\sigma=0.1$, the three curves nearly overlap for all values of $I$, as can be seen from Fig. 6(a). This is not surprising, as the initial conditions contain mostly $\mathrm{ABs}$ independent of the value of the correlation length. The spectra of eigenvalues in this case are almost identical. It is the MI that is driving the evolution when the perturbations are small. A higher level of perturbations transforms some ABs into solitons. The amplitudes of these solitons play a decisive role in increasing the field amplitudes. As can be seen from the eigenvalue sets, the higher $L_{c}$ creates solitons with higher amplitudes and consequently taller rogue waves. Figure 6(b), calculated for the case of $\sigma=0.5$, shows a higher tail of the PDF for the correlation length $L_{c}=0.76$ and even higher for $L_{c}=1.8$. This effect is more pronounced when $\sigma=0.9$, as shown in Fig. 6(c).

Soliton collisions are one of the major sources of rogue waves during supercontinuum generation in optical fibers [21]. This happens when more complicated physical effects are acting, such as third-order dispersion [22] and Raman effect [13]. The process involves cascades of soliton interactions with energy exchange between them before the most energetic solitons emerge at the fiber output [23,24]. The latter are known as optical rogue waves [25]. As we have found in the present work, the mechanism of rogue wave generation in an integrable system is different. It depends significantly on the relative number of breathers and solitons and their parameters.

In conclusion, we studied the process of rogue wave formation in a system governed by the NLSE. We used initial conditions with a variable random component and have shown that the increase of the probability of rogue wave generation is related to the relative content of solitons and breathers in the initial conditions. Our theory can be extended to other integrable models that have both solitons and breathers as essential parts of complex dynamics. These include vector NLSEs [26], three-wave parametric

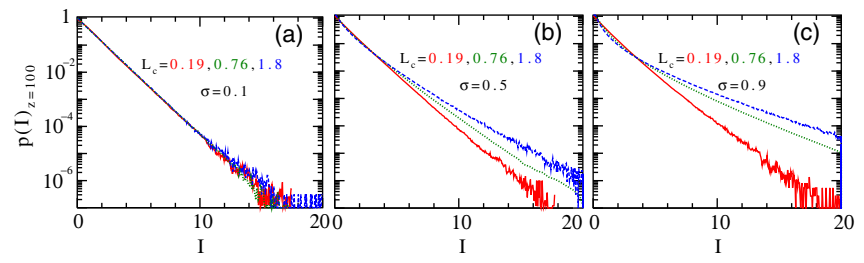

FIG. 6. (a) Probability density functions calculated at $\xi=100$ for three different values of $L_{c}$ and for (a) $\sigma=0.1$, (b) $\sigma=0.5$, and (c) $\sigma=0.9$. The solid red curves in each case correspond to $L_{c}=0.19$, the dotted green curve to $L_{c}=0.76$, and the dashed blue curve to $L_{c}=1.8$. 
interaction equations [27], and other similar models where rogue waves exist on an equal basis with solitons.

The authors acknowledge support from the Volkswagen Stiftung. The work of J. M. S.-C. was also supported by MINECO under Contract No. TEC2012-37958-C02-02, and by C. A. M. under Contract No. S2013/MIT-2790. N.D. and N. A. acknowledge the support of the ARC (Discovery Project No. DP140100265).

[1] G. K. Batchelor, The Theory of Homogeneous Turbulence (Cambridge University Press, Cambridge, England, 1953).

[2] S. Toenger, T. Godin, C. Billet, F. Dias, M. Erkintalo, G. Genty, and J. M. Dudley, Emergent rogue wave structures and statistics in spontaneous modulation instability, Sci. Rep. 5, 10380 (2015).

[3] P. Walczak, S. Randoux, and P. Suret, Optical Rogue Waves in Integrable Turbulence, Phys. Rev. Lett. 114, 143903 (2015).

[4] D. S. Agafontsev and V. E. Zakharov, Integrable turbulence and formation of rogue waves, Nonlinearity 28, 2791 (2015).

[5] K. Hammani, B. Kibler, C. Finot, and A. Picozzi, Emergence of rogue waves from optical turbulence, Phys. Lett. A 374, 3585 (2010).

[6] J. Garnier, M. Lisak, and A. Picozzi, Toward a wave turbulence formulation of statistical nonlinear optics, J. Opt. Soc. Am. B 29, 2229 (2012).

[7] A. Sauter, S. Pitois, G. Millot, and A. Picozzi, Incoherent modulation instability in instantaneous nonlinear Kerr media, Opt. Lett. 30, 2143 (2005).

[8] A. R. Osborne, Nonlinear Ocean Waves (Academic Press, New York, 2009).

[9] J. C. Wyngaard, Turbulence in the Atmosphere (Academic Press, New York, 2010).

[10] M. Vorontsov and J. Minet, in Proceedings of the Directed Energy Systems Symposium, Monterey, CA, 2013 (Directed Energy Professional Society (DEPS), Monterey, California, 2013).

[11] N. Akhmediev, J. M. Soto-Crespo, and A. Ankiewicz, How to excite a rogue wave, Phys. Rev. A 80, 043818 (2009).

[12] V. I. Shrira and V. V. Geogjaev, What makes the Peregrine soliton so special as a prototype of freak waves?, J. Eng. Math. 67, 11 (2010).
[13] J. M. Dudley, F. Dias, M. Erkintalo, and G. Genty, Instabilities, breathers and rogue waves in optics, Nat. Photonics 8, 755 (2014).

[14] V. E. Zakharov, N. Pushkarev, V. F. Shvets, and V. V. Yan'kov, Pis'ma Zh. Eksp. Teor. Fiz. 48, 79 (1988) [Soliton turbulence, JETP Lett. 48, 83 (1988)].

[15] N. Akhmediev, A. Ankiewicz, and J. M. Soto-Crespo, Rogue waves and rational solutions of the nonlinear Schrödinger equation, Phys. Rev. E 80, 026601 (2009).

[16] N. Akhmediev, A. Ankiewicz, J. M. Soto-Crespo, and J. M. Dudley, Universal triangular spectra in parametricallydriven systems, Phys. Lett. A 375, 775 (2011).

[17] N. Akhmediev, J. M. Soto-Crespo, and A. Ankiewicz, Extreme waves that appear from nowhere: On the nature of rogue waves, Phys. Lett. A 373, 2137 (2009).

[18] M. Rosenblatt, Gaussian and Non-Gaussian Linear Time Series and Random Fields (Springer-Verlag, New York, 2000).

[19] V.E. Zakharov and A. B. Shabat, Exact theory of twodimensional self-focusing and one-dimensional selfmodulation of waves in nonlinear media, J. Exp. Theor. Phys. 34, 62 (1972).

[20] Ch. Mahnke and F. Mitschke, Possibility of an Akhmediev breather decaying into solitons, Phys. Rev. A 85, 033808 (2012).

[21] J. M. Dudley, G. Genty, and S. Coen, Supercontinuum generation in photonic crystal fiber, Rev. Mod. Phys. 78, 1135 (2006).

[22] M. Taki, A. Mussot, A. Kudlinski, E. Louvergneaux, M. I. Kolobov, and M. Douay, Third-order dispersion for generating optical rogue solitons, Phys. Lett. A 374, 691 (2010).

[23] N. Akhmediev, J. M. Soto-Crespo, and A. Ankiewicz, Could rogue waves be used as efficient weapons against enemy ships?, Eur. Phys. J. Spec. Top. 185, 259 (2010).

[24] K. Hammani, B. Kibler, J. Fatome, S. Boscolo, G. Genty, J. M. Dudley, G. Millot, and Ch. Finot, Nonlinear spectral shaping and optical rogue events in fiber-based systems, Opt. Fiber Technol. 18, 248 (2012).

[25] D. Solli, C. Ropers, P. Koonath, and B. Jalali, Optical rogue waves, Nature (London) 450, 1054 (2007).

[26] B. Frisquet, B. Kibler, P. Morin, F. Baronio, M. Conforti, G. Millot, and S. Wabnitz, Optical dark rogue wave, Sci. Rep. 6, 20785 (2016).

[27] F. Baronio, M. Conforti, A. Degasperis, and S. Lombardo, Rogue Waves Emerging from the Resonant Interaction of Three Waves, Phys. Rev. Lett. 111, 114101 (2013). 